\title{
Topology Optimum Design of Piezoelectric Energy Harvesting Devices Using Two-Phase Piezoelectric Materials
}

\author{
Yingbing Wan, Li Zhang*, Xiaoming Qin, Liang Gao and Cong Jin \\ School of Mechanical Science and Engineering, Huazhong University of Science \& Technology, Wuhan, 430074, China \\ ${ }^{*}$ Corresponding author
}

\begin{abstract}
The paper presents a two-phase FEM-based topology optimization for piezoelectric energy harvesting devices. The goal of this research is to improve energy conversion efficiency of the piezoelectric cantilevered plates as an energy harvesting device based on the piezoelectric effect when applied to external loads. Owing to the friability of piezoelectric material, the piezoelectric energy harvesting devices can contain both piezoelectric material and elastic material. Therefore, an alternative way to express the piezoelectric material property interpolation for two-phase materials structure has been proposed. With this method, the structure composed of two materials, including elastic material and piezoelectric material, is regarded as the design variable under the topology optimization formulation. It seems to be a more reasonable solution for the topology optimization of piezoelectric composite materials. The method of moving asymptotes (MMA) is used to update the design variables. The energy conversion efficiency is compared using a developed FEM-based optimization code to verify the performance of the proposed method. Some numerical examples are presented and investigated to verify the availability of the proposed method.
\end{abstract}

Keywords-topology optimization; piezoelectric energy harvesting devices; two-phase material

\section{INTRODUCTION}

Converting the available energy from the environment allows a self-sufficient energy supply for low consumed electric loads such as wireless sensor networks or radio transmitters. Mechanical energy can be converted into electrical energy with the piezoelectric effect. Piezoelectric energy harvester can convert the kinetic energy from vibrations or shocks into electrical energy as presented in Sodano [1]. Piezoelectric materials can be divided in two main groups: crystals and ceramics. The most suitable piezoelectric material for energy harvester is lead zirconate titanate (PZT), macro fiber composite (MFC) and lead magnesium niobate/lead titanate (PMN-PT). Glynne-Jones P [2] focus on the design of piezoelectric energy harvester, using a two-phase topology optimization method which was proposed by Bendsøe M P, Sigmund O [3]. The goal is to maximize the mechanical to electrical energy conversation of the structure.

Recently, both foreign and domestic scholars have researched extensively about the topology optimization of piezoelectric devices. Martin Kogl and ECN Silva (2005)[4] proposed a new piezoelectric material model PEMAP-P (piezoelectric material with penalization and polarization) which is an extension of the SIMP (solid isotropic material with penalization). Jae Eun Kim [5] considered the effect of three different material coefficient groups, such as the mechanical, electrical, and electromechanical coefficient groups. The topology optimization of two-dimensional piezoelectric energy harvester was also studied. Dmitri Tcherniak [6] suggested the topology optimization of resonating structures to maximize the magnitude of steady-state vibrations for a given excitation frequency. Jin Yee Noh and Gil Ho Yoon [7] developed optimal layouts for piezoelectric energy harvesting devices by considering the harmonic dynamic coupling effects between the electric energy and an efficient structure. Bin Zheng [8] tried to study the energy harvesting devices using piezoelectric materials on the basis of a static case. The design objective of the devices is set to maximize the stored electric energy with both elastic materials and piezoelectric materials. Fabian Wein [9] investigated the vibrational piezoelectric energy harvesters in which an elastic beam is sandwiched between two piezoelectric plates and homogenize the piezoelectric strain distribution increasing the electric performance. Cheol Kim and Jinwoo Lee [10] proposed an efficient design method based on topology optimization coupled with Mindlin plate theory for designing the unimorph piezoelectric cantilevered vibrating electric harvester. S.L. Vatanabe [11] studied the influence of pattern gradation on the design of piezocomposite energy harvesting devices using topology optimization. Zhen Luo [12] presented a topology optimization method to design piezoelectric actuators using a multiphase level set method of piecewise constants.

According to the literature in recent years, various scholars examined the topology optimization of piezoelectric materials actuators and energy harvesters. Most of them use a topology optimization method that considers the distribution of one material phase. However, the piezoelectric energy harvesting cantilever combines a piezoelectric part and an elastic material part. Few investigations have been done on the topology optimization with both elastic material and piezoelectric material. Further studies are still essential. A topology optimization method considering the distribution of two materials has been proposed. We can use this method to develop a two-phase topology optimization method of piezoelectric energy harvester. 
Therefore, the purpose of this paper is to suggest a topology method for multiphysics and two-phase piezoelectric energy harvesting devices. A two-phase piezoelectric energy harvesting devices model is applied to interpolate the finite elements.The goal of this approach is to improve energy conversion efficiency of the piezoelectric cantilevered plates as an energy harvesting device based on the piezoelectric effect when applied to external loads. The topology optimization problem will be solved using MMA method.

This paper is organized as follows: a brief review of finite element model for the piezoelectric model is described in section 2, then a two-phase piezoelectric energy harvesting devices model and the problem formulation of optimization of the mechanical electrical energy conversion is detailed in section 3, the sensitivity of energy conversion is derived in section4. In section 5, two numerical examples are presented and investigated to verify the availability of the proposed method. Section 6 contains concluding remarks and recommendations for future work.

\section{PIEZOELECTRIC MATERIAL FINITE ELEMENT MODEL}

According to the coupling of mechanical and electrical fields for piezoelectric materials, the relationship between material strain and dielectric displacement under a certain condition can be expressed. We assumed that the changes of the electric field and mechanical stress are linear. The influence of temperature on the material properties is considered so small that we can ignore. With these assumptions in Benjeddou A [13] followed, the piezoelectric constitutive equation can be written as:

$$
\begin{gathered}
S_{1}=s_{11}^{E} T_{1}+d_{31} E_{3} \\
D_{3}=d_{31} T_{1}+\varepsilon_{33}^{\mathrm{T}} E_{3}
\end{gathered}
$$

Where $S, E, T$ and $D$ represents mechanical strain, electric field, mechanical stress and electric displacement, respectively. $s E, d, \varepsilon$ are mechanical elastic tensor matrix, piezoelectric constant matric and permittivity constant matrix, respectively.

Considering the constitutive relations above, the weak form of the piezoelectric constitutive equation can be written as Kim J E [5] :

$$
\begin{gathered}
\int_{\Omega} \delta u^{t} f_{v} d \Omega+\int_{A} \delta u^{t} f_{s} d A=\int_{\Omega} \delta S^{t} T d \Omega \\
=\int_{\Omega} \delta S^{t}\left(C^{E} \cdot S-e^{t} \cdot E\right) d \Omega \\
\int_{A} \delta \phi \cdot q d A=\int_{\Omega} \delta E^{t} D d \Omega=\int_{\Omega} \delta E^{t}\left(e \cdot S+\varepsilon^{S} \cdot E\right) d \Omega
\end{gathered}
$$

The symbols, $u$, $f v, f s$, represents displacement, body force and mechanical traction, and $\varphi$ and $q$ donates the electric potential and electric charge.
The finite element equations can be concluded from changing integration terms with $\mathrm{K}$ matricesin Zheng $\mathrm{B}$ (2009)[8]:

$$
\left[\begin{array}{cc}
K_{u u} & K_{u \phi} \\
K_{u \phi}^{t} & -K_{\phi \phi}
\end{array}\right]\left\{\begin{array}{l}
\tilde{u} \\
\tilde{\varphi}
\end{array}\right\}=\left\{\begin{array}{l}
f \\
q
\end{array}\right\}
$$

The symbols $K u u, K u \varphi, K \varphi \varphi$ denotes mechanical stiffness matrix, piezoelectric coupling matrix and dielectric matrix. $f$ and $q$ are the external node force and external node electric charges. $U$ and $\Phi$ are the nodal displacements vectors and electric potentials vectors. For piezoelectric energy harvester, these two vectors are unknown vectors. Certain static force and charge will be applied to the structure. After that, we can calculate the unknown nodal displacements and electric potentials. The definition of $K u u, K u \varphi, K \varphi \varphi$ in equation (5) is

$$
\begin{aligned}
& k_{u u}^{e}=\int_{\Omega_{e}}\left(B \cdot N_{u}\right)^{t} \cdot C^{E} \cdot\left(B \cdot N_{u}\right) d \Omega \\
& =\int_{\Omega_{e}} B_{u}{ }^{t} \cdot C^{E} \cdot B_{u} d \Omega,\left(B_{u}=B \cdot N_{u}\right) \\
& k_{\phi \phi}^{e}=\int_{\Omega_{e}}\left(\tilde{B} \cdot N_{\phi}\right)^{t} \cdot \varepsilon^{S} \cdot\left(\tilde{B} \cdot N_{\phi}\right) d \Omega \\
& =\int_{\Omega_{e}} \tilde{B}_{\phi}{ }^{t} \cdot \varepsilon^{S} \cdot \tilde{B}_{\phi} d \Omega,\left(\tilde{B}_{\phi}=\tilde{B} \cdot N_{\phi}\right) \\
& k_{u \phi}^{e}=\int_{\Omega_{e}}\left(B \cdot N_{u}\right)^{t} \cdot e \cdot\left(\tilde{B} \cdot N_{\phi}\right) d \Omega \\
& =\int_{\Omega_{e}} B_{u}{ }^{t} \cdot e \cdot \tilde{B}_{\phi} d \Omega
\end{aligned}
$$

$$
\begin{aligned}
& K_{u u}=\sum_{e} \int_{\Omega_{e}}\left(B \cdot N_{u}\right)^{t} \cdot C^{E} \cdot\left(B \cdot N_{u}\right) d \Omega=\sum_{e} k_{u u}^{e} \\
& K_{\phi \phi}=\sum_{e} \int_{\Omega_{e}}\left(\tilde{B} \cdot N_{\phi}\right)^{t} \cdot \varepsilon^{S} \cdot\left(\tilde{B} \cdot N_{\phi}\right) d \Omega=\sum_{e} k_{\phi \phi}^{e} \\
& K_{u \phi}=\sum_{e} \int_{\Omega_{e}}\left(B \cdot N_{u}\right)^{t} \cdot e \cdot\left(\tilde{B} \cdot N_{\phi}\right) d \Omega=\sum_{e} k_{u \phi}^{e}
\end{aligned}
$$

After the equilibrium represented above is established, Parameters needed in the next section can be computed. We will consider the topology optimization problem.

\section{FORMULATION OF OPTIMIZATION PROBLEM}

\section{A. Piezoelectric Material with Penalization Model}

In this section, we will study the formulation of piezoelectric energy harvester in topology optimization problem. First, a material model is needed to describe the distribution of the design area. In the early research, we use a SIMP model to describe the normal material. However, considering the piezoelectric effect, we will use the PEMAP-P model proposed by Silva E C N, Kikuchi N [14], which is an extension of the SIMP. This model parameterizes the material coefficient matrices as follows:

$$
\begin{aligned}
& C^{E}=x^{p} C_{0}^{E} \\
& \varepsilon^{S}=x^{p} \varepsilon_{0}{ }^{S} \\
& e=x^{p} e_{0}
\end{aligned}
$$


The symbols, $C^{E}, \varepsilon^{S}$, e, represents the stress strain relation under constant electrical field, the permittivity under constant strain and the piezoelectric coupling respectively.

\section{B. Two-Phase Piezoelectric Energy Harvesting Devices Model}

In the previous research, the piezoelectric energy harvesting cantilever combines a piezoelectric part and an elastic material part. Chen S.(2010)[15] suggested that if we only consider the distribution of one material, the result of optimization will not satisfy our requirement. Considering the distribution of two material phase in the design domain simultaneously is very necessary. In the following section, we will propose a new twophase piezoelectric energy harvesting devices model to interpolate the finite elements.

It is very important to choose an appropriate interpolation method to interpret intermediate element. The interpolation method must avoid the element that cannot be manufactured by materials. The two-phase piezoelectric model is an extension of the piezoelectric material with penalization and polarization model and multiple material phase model described by Sigmund O [16]. Considering the distribution of two materials in the design domain, the interpolation methods will be adjusted. A change of the power law approach is to express elasticity tensor, permittivity tensor and piezoelectric coupling tensor of elements as:

$$
\begin{aligned}
& C^{E}=x^{p c} C_{1}^{E}+(1-x)^{p c} C_{2}^{E} \\
& \varepsilon^{S}=x^{p s} \varepsilon_{1}^{S}+(1-x)^{p s} \varepsilon_{2}{ }^{S} \\
& e=x^{p e} e_{1}+(1-x)^{p e} e_{2}
\end{aligned}
$$

The symbols, $C_{1}{ }^{E}$ and $C_{2}{ }^{E}$ are the elasticity tensor of piezoelectric material and elastic material, respectively. $\varepsilon_{1}{ }_{1}$ and $\varepsilon_{2}^{S}$ are the permittivity tensor of piezoelectric material and elastic material. $e_{1}$ and $e_{2}$ are the piezoelectric coupling tensor of piezoelectric material and elastic material respectively. $x$ is the density design variable that is allocated to every finite element. $p$ is the penal factor.

\section{Optimization of the Mechanical Electrical Energy Conversion}

The energy conversion efficiency is the ratio between the electric output of an energy harvester and the mechanical input, in energy terms. It is an appropriate factor to measure the performance of a piezoelectric electric harvester. So, the energy conversion efficiency will be regarded as the objective function. As far as we know, the work done by external force stored in the form of strain energy is:

$$
W^{S}=u^{t} K_{u u} u
$$

The work stored in the form of electrical energy is:

$$
W^{S}=\varphi^{t} K_{\phi \phi} \varphi
$$

The topology optimization problem based on the power law approach, where the objective is to maximize energy conversion efficiency can be written as follows:

$$
\begin{aligned}
& \text { Minimize: } \varsigma_{1}=\frac{1}{\varsigma}=1+\frac{u^{t} K_{u u} u}{\varphi^{t} K_{\phi \phi} \varphi} \\
& \text { or Maximize: } \varsigma_{2}=\frac{\varphi^{t} K_{\phi \phi} \varphi}{u^{t} K_{u u} u} \\
& \text { Subject to: } \frac{V(\mathrm{x})}{V_{0}}=f \\
& {\left[\begin{array}{ll}
K_{u u} & K_{u \phi} \\
K_{u \phi}^{t} & -K_{\phi \phi}
\end{array}\right]\left\{\begin{array}{l}
u \\
\varphi
\end{array}\right\}=\left\{\begin{array}{l}
F \\
Q
\end{array}\right\}} \\
& 0<x_{\min } \leq x \leq 1
\end{aligned}
$$

Where $V(x)$ and $V_{0}$ are the piezoelectric material volume and design domain volume, respectively. $f$ is the prescribed volume fraction.

\section{SENSITIVITY ANALYSIS}

The topology optimization problem could be solved using several different approaches such as optimality criteria (OC) method which was proposed by Sigmund O [17], the method of moving asymptotes (MMA) which was developed by Svanberg K [18] and other methods. For the subject, we will use MMA method. It uses a special type of convex approximation and makes the solving of the complex objective function more effective. To renew the design variables using the method of moving asymptotes, sensitivity information about energy conversion efficiency and design constraints will be calculated. The sensitivity can be obtained by calculating the first order derivative of the object function. With the method illustrated in Zheng B et al[8], we can obtain the sensitivity of $\varsigma$ with respect to $x$ is:

$$
\frac{\partial \varsigma}{\partial x_{\mathrm{e}}}=\frac{1}{\Pi^{E}} \frac{\partial \Pi^{S}}{\partial x_{e}}-\frac{\Pi^{\mathrm{S}}}{\left(\Pi^{\mathrm{E}}\right)^{2}} \frac{\partial \Pi^{\mathrm{E}}}{\partial x_{e}}
$$

To avoid explicit calculations of the first order derivative of strain energy and electrical energy, we will use $\lambda$ and $\mu$ as the arbitrary adjoint displacement and potential vectors. After this method is called, the derivative of strain energy and electrical energy will be removed.

$$
\left[\begin{array}{cc}
K_{u u} & K_{u \phi} \\
K_{u \phi}^{t} & -K_{\phi \phi}
\end{array}\right]\left[\begin{array}{cc}
\lambda_{u} & \mu_{u} \\
\lambda_{\phi} & \mu_{\phi}
\end{array}\right]=\left[\begin{array}{cc}
-K_{u u} u & 0 \\
0 & -K_{\phi \phi} \varphi
\end{array}\right]
$$

The result can be substituted into object function. The sensitivity of energy conversion efficiency is: 


$$
\begin{aligned}
& \frac{\partial \varsigma}{\partial x_{\mathrm{e}}}=\frac{1}{\varphi^{t} K_{\phi \phi} \varphi}\left(\frac{1}{2} \frac{d f_{c}}{d x_{e}} u^{t} k_{u u}^{e} u+\frac{d f_{c}}{d x_{e}} \lambda_{u}^{t} k_{u u}^{e} u\right. \\
& \left.+\frac{d f_{c \varepsilon}}{d x_{e}} \lambda_{u}^{t} k_{u \phi}^{e} \varphi+\frac{d f_{c \varepsilon}}{d x_{e}} \lambda_{\phi}^{t} k_{\phi u}^{e} u-\frac{d f_{\varepsilon}}{d x_{e}} \lambda_{\phi}^{t} k_{\phi \phi}^{e} \varphi\right) \\
& -\frac{u^{t} K_{u u} u}{\left(\varphi^{t} K_{\phi \phi} \varphi\right)^{2}}\left(\frac{1}{2} \frac{d f_{\varepsilon}}{d x_{e}} \varphi^{t} k_{\phi \phi}^{e} \varphi+\frac{d f_{c}}{d x_{e}} \mu_{u}^{t} k_{u u}^{e} u\right. \\
& \left.+\frac{d f_{c \varepsilon}}{d x_{e}} \mu_{u}^{t} k_{u \phi}^{e} \varphi+\frac{d f_{c \varepsilon}}{d x_{e}} \mu_{\phi}^{t} k_{\phi u}^{e} u-\frac{d f_{\varepsilon}}{d x_{e}} u_{\phi}^{t} k_{\phi \phi}^{e} \varphi\right)
\end{aligned}
$$

\section{NUMERICAL EXAMPLES}

In this section, examples are presented to illustrate the result of the topology optimization method of piezoelectric energy harvesting devices. The design domain of these examples are all rectangles to simplify the calculation. The structure is fixed at the left, and a force is applied to it. The objective is to compute the optimal material distribution of the design domain. In addition, two material including a piezoelectric material and an elastic material is considered at the same time. In the example, Aluminum is used for elastic material, and PZT-5A is used for the piezoelectric material. The static force is applied to produce electricity by the deformation of the piezoelectric material. The iteration is terminated if the change in design variables is less than 0.01 . Otherwise, the calculations are repeated. All examples are calculated with MATLAB.

\section{A. Example 1}

The design domain is shown in Figure 1. The rectangle is the simplification of a unimorph cantilevered piezoelectric energy harvester with fixed boundary condition at left side. A static in-plane force of $100 \mathrm{~N}$ is applied at the middle of the right side boundary. The design domain is divided into $60 \times 30$ isotropic plate finite elements. The penal factor $p c, p s$, pe is 3, 4,6 , respectively. The volume constraint is set to $50 \%$. The elastic, piezoelectric, and dielectric coefficient matrices are set as follows:

$$
\begin{aligned}
{\left[\mathrm{C}^{E}\right] } & =\left[\begin{array}{cccccc}
12.7205 & 8.0212 & 8.4670 & 0 & 0 & 0 \\
8.0212 & 12.7205 & 8.4670 & 0 & 0 & 0 \\
8.4670 & 8.4670 & 11.7436 & 0 & 0 & 0 \\
0 & 0 & 0 & 2.2989 & 0 & 0 \\
0 & 0 & 0 & 0 & 2.2989 & 0 \\
0 & 0 & 0 & 0 & 0 & 2.3474
\end{array}\right] \times 10^{10} \\
{[\mathrm{e}] } & =\left[\begin{array}{cccccc}
0 & 0 & 0 & 0 & 17.0345 & 0 \\
0 & 0 & 0 & 17.0345 & 0 & 0 \\
-5.4000 & -5.4000 & 23.2403 & 0 & 0 & 0
\end{array}\right] \\
{\left[\varepsilon^{S}\right] } & =\left[\begin{array}{ccc}
1704 & 0 & 0 \\
0 & 1704 & 0 \\
0 & 0 & 1433.61
\end{array}\right]
\end{aligned}
$$

The polarization direction of the piezoelectric elements is applied from the bottom up. Two electrodes are put at the top and bottom surface of the design domain. But due to the thinness of the electrodes, the additional stiffness can be ignored in finite element analysis. The bottom electrode is connected to the ground. When the force applied to the piezoelectric harvester, strain will produced. And mechanical energy converts into electrical energy and strain energy by piezoelectric effect. After 167 times of iteration, the optimized structure parameters were obtained. The optimal result is shown in Figure 2 and the iteration curve of objective function is shown in Figure 3.

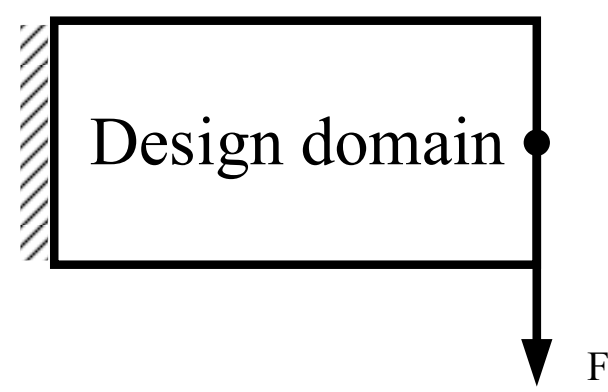

FIGURE I. THE DESIGN DOMAIN OF A TWO-PHASE MATERIAL ENERGY HARVESTER AND LOAD.

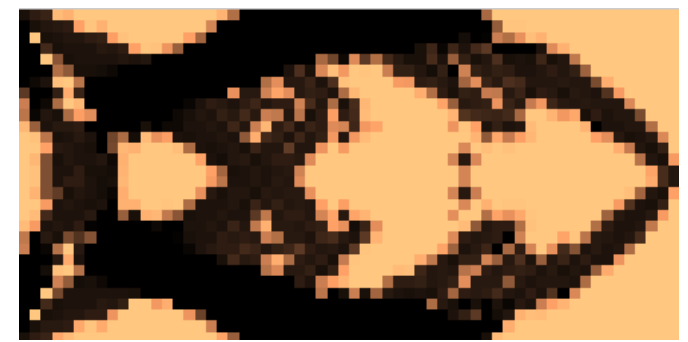

FIGURE II. THE OPTIMUM TOPOLOGY OF A PIEZOELECTRIC MATERIAL AND ALUMINUM SUBSTRATE

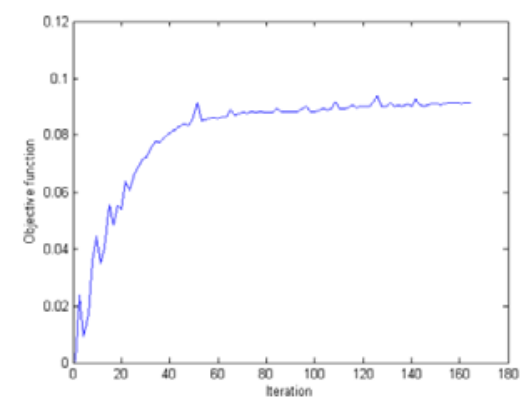

FIGURE III. ITERATION CURVE OF THE DESIGN OBJECTIVE FUNCTION

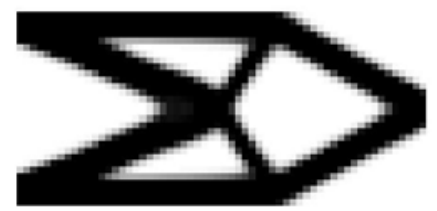

FIGURE IV. A SIMPLE CANTILEVER WHEN CONSIDERING MEAN COMPLIANCE ONLY. 
As is shown above, a new piezoelectric energy harvester has been designed. The black area in Figure 2 represents the piezoelectric material and the copper area represents the elastic material. The energy conversion efficiency of this model is $8.87 \%$. As a comparison, we calculate the energy conversion efficiency of a cantilever under the same loads when considering mean compliance only. The cantilever is mentioned in Sigmund $O[17]$. They aim to minimize the compliance of the in-plane cantilever, and the optimum design is shown in Figure 4. The reason we choose this one is that the finite element models of these cantilevers are similar. The material, volume constraint and other properties are the same in two calculation. After applying the computations, the energy conversion efficiency of a cantilever without optimization is $5.44 \%$. The optimized structure has $63.05 \%$ better performance than the design when considering mean compliance only. Compared to the optimized solution in which only piezoelectric material was considered by Zheng (2009)[8] whose improvement was only $34.4 \%$, our improvement is nearly two times as many as theirs. Through comparison, the result shows that an optimized cantilever has an improvement in energy harvesting.

\section{B. Example 2}

This example discusses the effects of the direction of applying force. The model is also an in-plane piezoelectric energy harvester under a static force. Contrary to the earlier example, the force applied on the structure is toward the right, as shown in Figure 5. As with the last example, the design domain is divided into $60 \times 30$ isotropic plate finite elements. Most of the properties are same as example 1.

The optimum distribution of the material is shown in Figure 7 , and the iteration curve of the objective function is shown in Figure 8. The energy conversion efficiency is $1.83 \%$. Meanwhile, we calculate the simple cantilever under the same condition when considering compliance only, and the optimum design is shown in Figure 6. The energy conversion efficiency is $1.72 \%$. The efficiency improvement is $6.40 \%$. It is far less than the last example. Analysis results show that the direction of external force acting has a significant effect on the performance of the optimal results and the angle between the polarization direction of the piezoelectric element and external force is important, too.

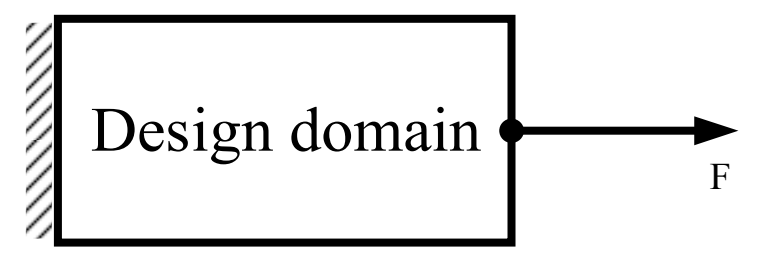

FIGURE V. THE DESIGN DOMAIN OF A TWO-PHASE MATERIAL ENERGY HARVESTER AND LOAD IN EXAMPLE 2.

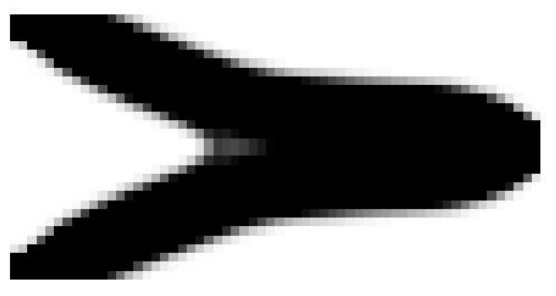

FIGURE VI. A SIMPLE CANTILEVER UNDER SAME LOADS WHEN CONSIDERING MEAN CONPLIANCE ONLY IN EXAMPLE 2.

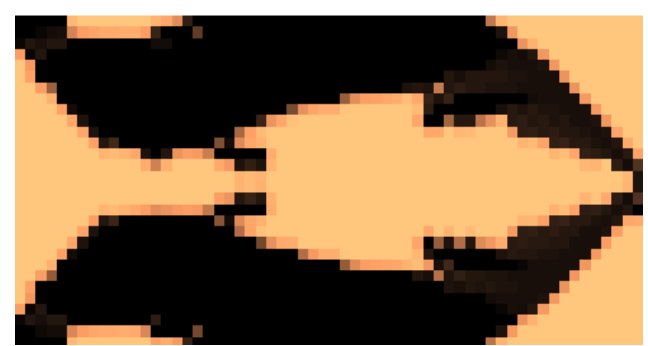

FIGURE VII. THE OPTIMUM TOPOLOGY OF A PIEZOELECTRIC MATERIAL AND ALUMINUM SUBSTRATE IN EXAMPLE 2.

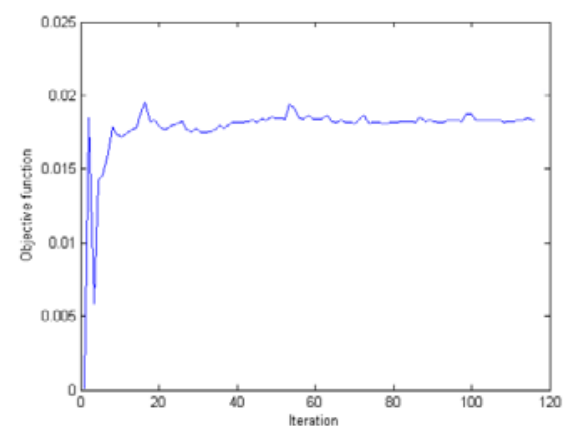

FIGURE VIII. ITERATION CURVE OF THE DESIGN OBJECTIVE FUNCTION IN EXAMPLE 2.

\section{CONCLUSIONS}

This paper has presented a topology optimization method for two-phase piezoelectric energy harvesting devices. The study focus on improving the efficiency of piezoelectric energy harvesting devices based on the piezoelectric effect when applied to external loads. The model comprises two parts: piezoelectric material and elastic material. A two-phase piezoelectric energy harvesting devices model is proposed to interpolate the finite elements, and then the energy conversion efficiency is compared using a developed FEM-based optimization code to prove the proposed method above. Examples under different load cases are presented. When the direction of applying force is proper, the optimized structure has better performance than the design without optimization. Some further illumination for the multi-phase material of 
piezoelectric energy harvesting devices can be learned from above design examples.

\section{ACKNOWLEDGMENT}

This research was sponsored in part by National Natural Science Foundation of China (Grant No: 51405170) and The National High-Tech Research and Development Program (863 Program) of China 2015AA042505.

\section{REFERENCES}

[1] Sodano H A, Inman D J, Park G. Comparison of piezoelectric energy harvesting devices for recharging batteries[J]. Journal of Intelligent Material Systems and Structures, 2005, 16(10): 799-807.

[2] Glynne-Jones P, Beeby S P, White N M. Towards a piezoelectric vibration-powered microgenerator[J]. IEE Proceedings-Science, measurement and technology, 2001, 148(2): 68-72.

[3] Bendsøe M P, Sigmund O. Topology optimization: theory, methods and applications. 2003[J].

[4] Kögl M, Silva E C N. Topology optimization of smart structures: design of piezoelectric plate and shell actuators [J]. Smart Materials and Structures, 2005, 14(2): 387.

[5] Kim J E, Kim D S, Ma P S, et al. Multi-physics interpolation for the topology optimization of piezoelectric systems [J]. Computer Methods in Applied Mechanics and Engineering, 2010, 199(49): 3153-3168.

[6] Tcherniak D. Topology optimization of resonating structures using SIMP method [J]. International Journal for Numerical Methods in Engineering, 2002, 54(11): 1605-1622.

[7] Noh J Y, Yoon G H. Topology optimization of piezoelectric energy harvesting devices considering static and harmonic dynamic loads[J]. Advances in Engineering Software, 2012, 53: 45-60.

[8] Zheng B, Chang C J, Gea H C. Topology optimization of energy harvesting devices using piezoelectric materials[J]. Structural and Multidisciplinary Optimization, 2009, 38(1): 17-23.

[9] Wein F, Kaltenbacher M, Stingl M. Topology optimization of a cantilevered piezoelectric energy harvester using stress norm constraints[J]. Structural and Multidisciplinary Optimization, 2013, 48(1): 173-185.

[10] Kim C, Lee J. Topology optimum design of unimorph piezoelectric cantilevered Mindlin plates as a vibrating electric harvester[J]. Journal of Mechanical Science and Technology, 2014, 28(10): 4131-4138.

[11] Vatanabe S L, Paulino G H, Silva E C N. Influence of pattern gradation on the design of piezocomposite energy harvesting devices using topology optimization[J]. Composites Part B: Engineering, 2012, 43(6): 2646-2654.

[12] Luo Z, Wang M Y, Wang S, et al. A level set - based parameterization method for structural shape and topology optimization[J]. International Journal for Numerical Methods in Engineering, 2008, 76(1): 1-26.

[13] Benjeddou A. Advances in piezoelectric finite element modeling of adaptive structural elements: a survey[J]. Computers \& Structures, 2000 76(1): 347-363.

[14] Silva E C N, Kikuchi N. Design of piezoelectric transducers using topology optimization[J]. Smart Materials and Structures, 1999, 8(3): 350 .

[15] Chen S, Gonella S, Chen W, et al. A level set approach for optimal design of smart energy harvesters[J]. Computer Methods in Applied Mechanics and Engineering, 2010, 199(37): 2532-2543.

[16] Sigmund O. Design of multiphysics actuators using topology optimization-Part II: Two-material structures[J]. Computer methods in applied mechanics and engineering, 2001, 190(49): 6605-6627.

[17] Sigmund O. A 99 line topology optimization code written in Matlab[J] Structural and multidisciplinary optimization, 2001, 21(2): 120-127.

[18] Svanberg K. The method of moving asymptotes - a new method for structural optimization[J]. International journal for numerical methods in engineering, 1987, 24(2): 359-373. 